\title{
Optimal Controller Initialization for Switching between Stabilizing Controllers
}

\author{
João P. Hespanha, Paolo Santesso, Gregory Stewart
}

\begin{abstract}
An important class of switched systems consists of those systems built as the feedback interconnection of a plant to be controlled, together with a multicontroller whose dynamics switch. When designing this type of switching controllers, one is faced with the questions of how to select controller realizations and how to initialize the state of the controllers as they are switched into the feedback loop. This paper deals with these questions for the problem of switching between linear timeinvariant controllers. We show that significant improvements in closed-loop performance can be attained by optimally choosing the initial state of a controller, as this controller is switched into the feedback loop. By including appropriate constraints on the optimizations used to select controller initializations, we show that the resulting switched closed-loop can be made uniformly input-to-state stable for arbitrary switching. Simulations illustrate the benefits of our approach with respect to others previously suggested in the literature.
\end{abstract}

\section{INTRODUCTION}

When dealing with the control of complex systems, multiple conflicting requirements on the closed-loop system often make a single linear time-invariant (LTI) controller unsuitable [1]. In this context, a convenient solution consists of designing several LTI controllers with transfer functions $\left\{k_{p}(s): p \in \mathcal{P}\right\}$, each one of them designed to meet only some specifications, and then switching between them in order to achieve the best overall performance [2]-[5]. This paper does not address the problem of how to select a "suitable" switching sequence, but instead is focused on how to guarantees stability of the switched closed-loop and how to obtain the best possible performance for an externally given desired sequence of controllers.

The switched systems considered here arise from the feedback interconnection of a plant $\Sigma$ to be controlled with a multicontroller $\mathbf{C}(\sigma)$ whose inputs are the usual tracking error $e_{T}(t)$ as well as a piecewise constant switching signal $\sigma:[0,+\infty) \rightarrow \mathcal{P}$ that roughly determines which should be the controller transfer function at time $t$ (cf. Figure 1). In particular, given $n$-dimensional state space realizations $E_{p}, F_{p}, G_{p}, H_{p}$ for each $k_{p}(s), p \in \mathcal{P}$, the state $x_{\text {mult }}(t)$

This material in this paper is based upon work supported by the Fondazione Aldo Gini and by the National Science Foundation under Grant No. CCR-0311084.

J. Hespanha is with the Dept. of Electrical and Computer Eng., Univ. of California, Santa Barbara, CA 93106-9560, USA hespanhadece.ucsb.edu; P. Santesso is with the Dept. of Information Eng., Univ. of Padova, Italy santesso@dei.unipd.it; and G. Stewart is with Honeywell, 500 Brooksbank Avenue, North Vancouver, BC, Canada, V7J 3S4 greg.stewart @honeywell.com

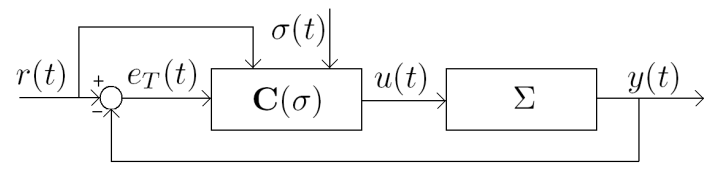

Fig. 1. Controller architecture

of the multicontroller $\mathbf{C}(\sigma)$ evolves according to

$$
\left\{\begin{array}{l}
\dot{x}_{\text {mult }}(t)=E_{\sigma(t)} x_{\text {mult }}(t)+F_{\sigma(t)} e_{T}(t) \\
u(t)=G_{\sigma(t)} x_{\text {mult }}(t)+H_{\sigma(t)} e_{T}(t)
\end{array}\right.
$$

on any time interval on which the switching signal $\sigma(t)$ remains constant, and according to

$$
x_{\text {mult }}(t)=F\left(x_{\text {mult }}\left(t^{-}\right), \sigma\left(t^{-}\right), \sigma(t), r(t)\right),
$$

at every time $t$, called a switching time, at which $\sigma(t)$ is discontinuous. The function $F(\cdot)$ is called the reset map and, given a signal $z(t)$, we denote by $z\left(t^{-}\right)$the limit from the left $\lim _{\tau \uparrow t} z(\tau)$. All signals are assumed continuous from the right. One should emphasize that even if each $k_{p}(s)$ stabilizes the process, as the value of $\sigma(t)$ switches within $\mathcal{P}$, the stability of the closed loop may be lost [3], [5].

A wide body of literature is available on conditions for the uniform stability of switched systems [5]-[8] and on the optimal control of such systems [9]-[14]. However, the special structure given by the feedback interconnection of a multicontroller with a non-switching plant provides a special structure that is usually not taken into account in the previously mentioned works.

Besides the choice of the controller transfer functions $k_{p}(s)$ and the selection of the switching signal $\sigma(t)$, there are two additional degrees of freedom available to the designer of the multi-controller: the selection of state space realizations for each controller and the construction of the reset map. A study of how these choices affect system stability appeared in [3], which addressed the problem of finding realizations and reset maps for a given family of stabilizing controller transfer functions such that the closed-loop system remains uniformly stable for every switching signal $\sigma(t)$. However, [3] does not explore the performance implications of the controller realizations and reset maps. In addition, this reference only considers very special types of reset maps.

The more recent paper [4] considers a similar setup and suggests the creation of several "candidate" control signals $v_{p}(t), p \in \mathcal{P}$, one for each controller, by letting each individual controller evolve continuously without resets. Then the 
switching signal $\sigma(t)$ selects which one among the $v_{p}(t)$ 's should be actually employed for control purpose. In order to get a smoother transient response, the piecewise continuous signal $v(t):=v_{\sigma(t)}(t)$ thus obtained is filtered in order to generate a continuous control input $u(t)$.

Inspired by the control scheme proposed in [3], we deal with the issue of appropriately designing the reset map, so that the closed-loop switched system produces transients that minimize a given cost function, while preserving the (input-to-state) stability of the closed-loop switched system. Simulation results compare the performance of our switching controller with those in [3] and [4].

\section{BACKGROUND AND PROBLEM FORMULATION}

Consider an LTI process $\Sigma$ with transfer function $g(s)$ from the input $u(t)$ to the output $y(t)$, and assume given a finite family of controller transfer functions $\left\{k_{p}(s): p \in \mathcal{P}\right\}$ from the tracking error $e_{T}(t):=r(t)-y(t)$ to the control input $u(t)$, where $r(t)$ denotes a reference signal.

For simplicity we restrict our attention to asymptotically stable single input single output (SISO) processes $\Sigma$. However, all the results presented here could be generalized to not necessarily stable multiple-input, multiple-output processes (MIMO) processes, following the approach in [3].

The construction of the multicontroller follows [3] and is inspired by the Youla-Kucera parametrization of all the stabilizing controllers. This parameterization motivates us to express each controller transfer function as

$$
k_{p}(s)=\frac{q_{p}(s)}{1-q_{p}(s) g(s)},
$$

which can be viewed as a positive feedback interconnection between a system with transfer function $g(s)$ and an asymptotically stable system with transfer function

$$
q_{p}(s)=\frac{k_{p}(s)}{1+g(s) k_{p}(s)} .
$$

Note that $q_{p}(s)$ is indeed asymptotically stable because $k_{p}(s)$ stabilizes $g(s)$ (see Figure 2).

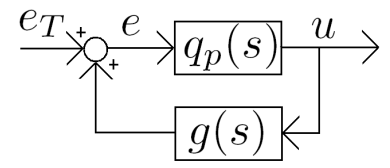

Fig. 2. Controller $k_{p}(s)$

Let $(A, B, C)$ denote a stabilizable and detectable $n_{\mathrm{pl}^{-}}$ dimensional realization for the process transfer function $g(s)$ and select stabilizable and detectable realizations $\left(A_{p}, B_{p}, C_{p}, D_{p}\right)$ for each $q_{p}(s), p \in \mathcal{P}$. The (not necessarily minimal) realizations for all the $q_{p}(s)$ should have the same dimension $n_{\mathrm{cn}}$ and all the Hurwitz matrices $A_{p}$ should admit the squared-norm of the state as a Lyapunov function, which is to say that

$$
A_{p}+A_{p}^{\prime}<0, \quad \forall p \in \mathcal{P}
$$

This is always possible in view of [3, Lemma 7]. In this paper, given a matrix $M$, we denote its transpose by $M^{\prime}$. In view of the diagram in Figure 2, each transfer function $k_{p}(s)$ has a realization $\left(E_{p}, F_{p}, G_{p}, H_{p}\right)$ with

$$
\begin{array}{rlrl}
E_{p} & :=\left[\begin{array}{cc}
A_{p} & B_{p} C \\
B C_{p} & A+B D_{p} C
\end{array}\right], & & F_{p}:=\left[\begin{array}{c}
B_{p} \\
B D_{p}
\end{array}\right], \\
G_{p}:=\left[\begin{array}{ll}
C_{p} D_{p} C
\end{array}\right], & H_{p}:=D_{p} .
\end{array}
$$

The proposed multicontroller $\mathbf{C}_{\sigma}$ has state $x_{\text {mult }}(t):=$ $\left[x_{\mathrm{cn}}(t)^{\prime} x_{\text {copy }}(t)^{\prime}\right]^{\prime}$, which evolves according to (1) on any time interval on which $\sigma(t)$ remains constant and

$$
x_{\text {mult }}(t)=\left[\begin{array}{c}
x_{\mathrm{cn}}(t) \\
x_{\mathrm{copy}}(t)
\end{array}\right]=\left[\begin{array}{c}
F\left(x_{\mathrm{mult}}\left(t^{-}\right), \sigma\left(t^{-}\right), \sigma(t), r(t)\right) \\
x_{\mathrm{copy}}\left(t^{-}\right)
\end{array}\right]
$$

at every switching time $t$. Note that (7) slightly differs from (2), since in the former the reset map $F(\cdot)$ only affects the component $x_{\mathrm{cn}}$ of the state of $\mathbf{C}(\sigma)$. In (7), the component $x_{\text {copy }}$ of the state of $\mathbf{C}(\sigma)$ remains continuous and it will actually converge to the process state $x_{\mathrm{pl}}(t)$. In fact, it follows from (1) and (7) that

$$
\dot{x}_{\text {copy }}(t)=A x_{\text {copy }}(t)+B u(t)
$$

for all times and, because we are assuming that $A$ is asymptotically stable, we indeed have that $x_{\text {copy }}(t)$ converges to $x_{\mathrm{pl}}(t)$, regardless of the control signal $u(t)$.

Connecting the plant $\Sigma$ with realization $(A, B, C)$ with the multicontroller $\mathbf{C}(\sigma)$, through the negative feedback interconnection in Figure 1, results in a switched system with a state $x(t):=\left[x_{\mathrm{pl}}(t)^{\prime} x_{\mathrm{cn}}(t)^{\prime} x_{\mathrm{copy}}(t)^{\prime}\right]^{\prime}$ that evolves according to

$$
\dot{x}(t)=\hat{A}_{\sigma(t)} x(t)+\hat{B}_{\sigma(t)} r(t), \quad y(t)=\hat{C}_{\sigma(t)} x(t)
$$

on any time interval on which $\sigma(t)$ remains constant and

$$
x(t)=\left[\begin{array}{c}
x_{\mathrm{pl}}(t) \\
x_{\mathrm{cn}}(t) \\
x_{\mathrm{copy}}(t)
\end{array}\right]=\left[\begin{array}{c}
x_{\mathrm{pl}}\left(t^{-}\right) \\
F\left(x\left(t^{-}\right), \sigma\left(t^{-}\right), \sigma(t), r(t)\right) \\
x_{\mathrm{copy}}\left(t^{-}\right)
\end{array}\right]
$$

at every switching time $t$. The matrices in (9) are defined by

$$
\begin{aligned}
\hat{A}_{p} & :=\left[\begin{array}{ccc}
A & B C_{p} & B D_{p} C \\
-B_{p} C & A_{p} & B_{p} C \\
-B D_{p} C & B C_{p} & A+B D_{p} C
\end{array}\right], \quad \hat{B}_{p}:=\left[\begin{array}{c}
B D_{p} \\
B_{p} \\
B D_{p}
\end{array}\right] \\
\hat{C}_{p} & :=\left[\begin{array}{lll}
C & 0 & 0
\end{array}\right], \quad \forall p \in \mathcal{P} .
\end{aligned}
$$

with all the $\hat{A}_{p}$ Hurwitz, since $k_{p}(s)$ stabilizes $g(s)$.

The remainder of this paper is focused on the goal of selecting an appropriate reset map $F(\cdot)$ that achieves optimal transient performance at switching times, while maintaining (9)-(10) stable. The issue of optimizing transient performance is the subject of the next section.

\section{AN OPTIMAL DEFINITION OF THE RESET MAP}

Suppose that at a time $t=t_{0}$ the switching signal jumps from $\sigma\left(t_{0}^{-}\right)=p$ to $\sigma\left(t_{0}\right)=q$. Our goal is to select the post-switching state defined by the reset map

$$
x_{\mathrm{cn}}\left(t_{0}\right)=F\left(x_{\text {mult }}\left(t_{0}^{-}\right), p, q, r\left(t_{0}\right)\right)
$$

so as to optimize the resulting transient performance, as measured by a quadratic cost of the following form 


$$
\begin{array}{r}
J=\int_{t_{0}}^{t_{1}}\left(e_{T}(t)^{\prime} R_{T}(t)+\dot{y}(t)^{\prime} W \dot{y}(t)+u(t)^{\prime} K u(t)\right) d t \\
+\left(x\left(t_{1}\right)-x_{\infty}\right)^{\prime} T\left(x\left(t_{1}\right)-x_{\infty}\right) \quad(12)
\end{array}
$$

where $R, W, K, T$ are appropriately selected symmetric positive semi-definite matrices and

$$
x_{\infty}:=-\hat{A}_{q}^{-1} \hat{B}_{q} r\left(t_{0}\right) .
$$

The choice of the matrices $R, W, K, T$ allows one to penalize the tracking error magnitude, output rate of change, control effort, and final state magnitude, respectively. In performing this optimization, it will be assumed that the switching signal $\sigma(t)$ and the reference $r(t)$ remain constant equal to $q$ and $r\left(t_{0}\right)$, respectively, over the optimization horizon $\left[t_{0}, t_{1}\right]$. If $\sigma(t)$ turns out to switch again before $t_{1}$, the value to which $x_{\mathrm{cn}}$ was reset at time $t_{0}$, will generally not be optimal. However, we will later make sure that even in this case, stability is guaranteed. Note that the vector $x_{\infty}$ that appears in the terminal term in (12) is the steady-state value to which $x(t)$ would converge as $t \rightarrow \infty$ if both $\sigma(t)$ and $r(t)$ were to remain constant.

\section{A. Optimization of transient performance}

To find the value of $x_{\mathrm{cn}}\left(t_{0}\right)$ that minimizes (12) we need to introduce some notation: Let $Q_{q}$ denote the symmetric solution to the following Lyapunov equation

$$
Q_{q} \hat{A}_{q}+\hat{A}_{q}^{\prime} Q_{q}=-P_{q},
$$

where $P_{q}:=\hat{C}_{q}^{\prime} R \hat{C}_{q}+\hat{A}_{q}^{\prime} \hat{C}_{q}^{\prime} W \hat{C}_{q} \hat{A}_{q}+\tilde{C}_{q}^{\prime} K \tilde{C}_{q} \geq 0$ and $\tilde{C}_{q}:=\left[\begin{array}{lll}-D_{q} C & C_{q} D_{q} C\end{array}\right]$. Such solution exists and is at least positive semi-definite because $\hat{A}_{q}$ is a Hurwitz matrix.

Let $\Delta:=t_{1}-t_{0}$ and introduce the positive semi-definite matrix $M_{q}:=Q_{q}-e^{\hat{A}_{q}^{\prime} \Delta}\left(Q_{q}+T\right) e^{\hat{A}_{q} \Delta}$ and the vector

$$
\begin{aligned}
g_{q}^{\prime}:= & 2 r\left(t_{0}\right)^{\prime}\left(\left(-R \hat{C}_{q}+\hat{B}_{q}^{\prime} \hat{C}_{q}^{\prime} W \hat{C}_{q} \hat{A}_{q}+D_{q}^{\prime} K \tilde{C}_{q}+\right.\right. \\
& \left.+Q_{q} \hat{B}_{q}^{\prime} Q_{q}\right)\left(I-e^{\hat{A}_{q} \Delta}\right) \hat{A}_{q}^{-1}+ \\
& \left.+\hat{B}_{q}^{\prime}\left(e^{\hat{A}_{q}^{\prime} \Delta}\left(\hat{A}_{q}^{\prime}\right)^{-1}\left(Q_{q}-T\right)-\left(\hat{A}_{q}^{\prime}\right)^{-1} Q_{q}\right) e^{\hat{A}_{q} \Delta}\right) .
\end{aligned}
$$

We will further need to block-partition the symmetric matrices $M_{q}$ and the vectors $g_{q}$ according to the partition in (10) of the state vector:

$$
M_{q}=\left[\begin{array}{lll}
M_{11}^{q} & M_{12}^{q} & M_{13}^{q} \\
M_{21}^{q} & M_{22}^{q} & M_{23}^{q} \\
M_{31}^{q} & M_{32}^{q} & M_{33}^{q}
\end{array}\right], \quad \quad g_{q}=\left[\begin{array}{c}
g_{1}^{q} \\
g_{2}^{q} \\
g_{3}^{q}
\end{array}\right]
$$

and perform a singular value decomposition of

$$
M_{22}^{q}=\left[\begin{array}{ll}
U_{1}^{q} U_{2}^{q}
\end{array}\right]\left[\begin{array}{cc}
\Lambda_{q} & 0 \\
0 & 0
\end{array}\right]\left[\begin{array}{l}
\left(V_{1}^{q}\right)^{\prime} \\
\left(V_{2}^{q}\right)^{\prime}
\end{array}\right]
$$

(with $\Lambda_{q}$ nonsingular). We are now ready to provide the solution to the minimization problem considered above.

Theorem 1: Assuming that $\sigma(t)=q$ and $r(t)=r\left(t_{0}\right)$, $\forall t \in\left[t_{0}, t_{1}\right]$, the global minimum to (12) with smallest norm is given by

$$
\begin{aligned}
x_{\mathrm{cn}}^{*}\left(t_{0}\right) & =V_{1}^{q} \Lambda_{q}^{-1}\left(U_{1}^{q}\right)^{\prime} . \\
& \left(\frac{1}{2} g_{2}^{q}-\left(\left(M_{12}^{q}\right)^{\prime} x_{\mathrm{pl}}\left(t_{0}\right)+M_{23}^{q} x_{\mathrm{copy}}\left(t_{0}\right)\right)\right) .
\end{aligned}
$$

Figure 3 depicts the result of numerical simulations illustrating how varying the length of the optimization interval may influence the system's behavior. It generally happens that the transient response improves as we increase the optimization interval. It should be noted that all the results in this section hold for an infinite horizon, as we make $t_{1} \rightarrow \infty$ in (12), in which case all the matrix exponentials $e^{\hat{A}_{q} \Delta}$ above become the zero matrix.

Proof of Theorem 1. We start by computing the criterion $J$ in (12) along a solution

$$
\begin{aligned}
& x(t)=e^{\hat{A}_{q}\left(t-t_{0}\right)} x\left(t_{0}\right)+\int_{t_{0}}^{t} e^{\hat{A}_{q}(t-\tau)} \hat{B}_{q} \cdot r\left(t_{0}\right) d \tau, \\
& u(t)=\tilde{C}_{q} x(t)+D_{q} r(t),
\end{aligned}
$$

$\forall t \in\left[t_{0}, t_{1}\right]$. In what follow, $*$ stands for additive terms that does not depend on the value of $x_{\mathrm{cn}}\left(t_{0}\right)$. Straightforward algebra shows that the terminal term in (12) is given by

$$
\begin{aligned}
\left(x\left(t_{1}\right)-\right. & \left.x_{\infty}\right)^{\prime} T\left(x\left(t_{1}\right)-x_{\infty}\right) \\
= & x\left(t_{0}\right)^{\prime} e^{\hat{A}_{q}^{\prime} \Delta} T e^{\hat{A}_{q} \Delta} x\left(t_{0}\right)+ \\
& +2 r\left(t_{0}\right)^{\prime} \hat{B}_{q}^{\prime} e^{\hat{A}_{q}^{\prime} \Delta}\left(\hat{A}_{q}^{\prime}\right)^{-1} T e^{\hat{A}_{q} \Delta} x\left(t_{0}\right)+*
\end{aligned}
$$

and that the integral term in (12) is given by

$$
\begin{gathered}
\int_{t_{0}}^{t_{1}}\left(e_{T}(t)^{\prime} R e_{T}(t)+\dot{y}(t)^{\prime} W \dot{y}(t)+u(t)^{\prime} K u(t)\right) d t \\
=\int_{t_{0}}^{t_{1}}\left(r\left(t_{0}\right)^{\prime}\left(R+\hat{B}_{q}^{\prime} \hat{C}_{q}^{\prime} W \hat{C}_{q} \hat{B}_{q}+D_{q}^{\prime} K D_{q}\right) r\left(t_{0}\right)\right. \\
\left.+x(t)^{\prime} P_{q} x(t)+c_{q}^{\prime} x(t)\right) d t+* \\
=\int_{t_{0}}^{t_{1}}\left(x(t)^{\prime} P_{q} x(t)+c_{q}^{\prime} x(t)\right) d t+*
\end{gathered}
$$

where $P_{q}$ has been already defined and $c_{q}^{\prime}=$ $2 r\left(t_{0}\right)^{\prime}\left(-R \hat{C}_{q}+\hat{B}_{q}^{\prime} \hat{C}_{q}^{\prime} W \hat{C}_{q} \hat{A}_{q}+D_{q}^{\prime} K \tilde{C}_{q}\right)$. The computational of the integral in (17) is fairly standard:

$$
\begin{gathered}
\int_{t_{0}}^{t_{1}}\left(x(t)^{\prime} P_{q} x(t)+c_{q}^{\prime} x(t)\right) d t \\
=\int_{t_{0}}^{t_{1}}\left(2 r\left(t_{0}\right)^{\prime} \hat{B}_{q}^{\prime} Q_{q} x(t)-\frac{d}{d t}\left(x^{\prime} Q_{q} x\right)+c_{q}^{\prime} x(t)\right) d t \\
=\int_{t_{0}}^{t_{1}}\left(2 r\left(t_{0}\right)^{\prime} \hat{B}_{q}^{\prime} Q_{q}+c_{q}^{\prime}\right) x(t) d t-\left.x^{\prime} Q_{q} x\right|_{t_{0}} ^{t_{1}} \\
=\int_{t_{0}}^{t_{1}}\left(2 r\left(t_{0}\right)^{\prime} \hat{B}_{q}^{\prime} Q_{q}+c_{q}^{\prime}\right) e^{\hat{A}_{q}\left(t-t_{0}\right)} x\left(t_{0}\right) d t-\left.x^{\prime} Q_{q} x\right|_{t_{0}} ^{t_{1}}+* \\
=x\left(t_{0}\right)^{\prime}\left(Q_{q}-e^{\hat{A}_{q}^{\prime} \Delta} Q_{q} e^{\hat{A}_{q} \Delta}\right) x\left(t_{0}\right)- \\
\quad\left(\left(c_{q}^{\prime}+2 r\left(t_{0}\right)^{\prime} Q_{q} \hat{B}_{q}^{\prime} Q_{q}\right)\left(I-e^{\hat{A}_{q} \Delta}\right) \hat{A}_{q}^{-1}\right. \\
\left.+2 r\left(t_{0}\right)^{\prime} \hat{B}_{q}^{\prime}\left(e^{\hat{A}_{q}^{\prime} \Delta}-I\right)\left(\hat{A}_{q}^{\prime}\right)^{-1} Q_{q} e^{\hat{A}_{q} \Delta}\right) x\left(t_{0}\right)+*
\end{gathered}
$$

Combining (16) and (18), we conclude that

$$
J=x\left(t_{0}\right)^{\prime} M_{q} x\left(t_{0}\right)-x\left(t_{0}\right)^{\prime} g_{q}+*,
$$




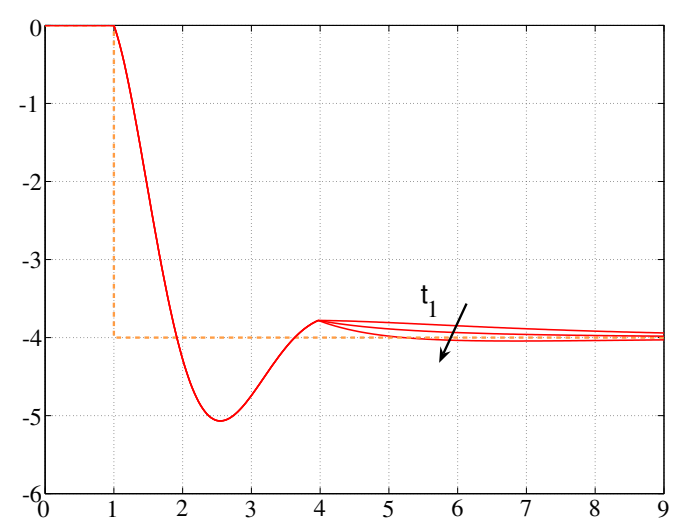

(a) $R=I, W \neq 0, K, T=0$

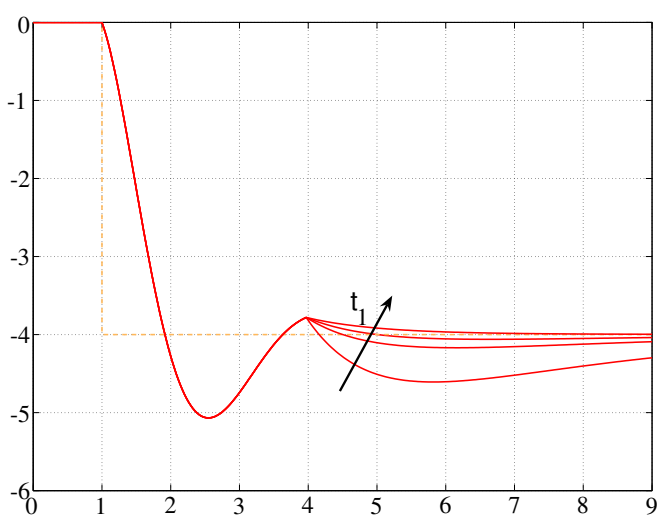

(b) $R=I, W, K, T=0$

Fig. 3. Transient responses for different weighting matrices and different durations for the optimization interval $\left[t_{0}, t_{1}\right]$. Details on the process and controllers being switched can be found in Section VI. In both plots there is a single controller switching at time $t_{0}=4$ sec.

but since our optimization is only performed on the component $x_{\mathrm{cn}}\left(t_{0}\right)$ of $x\left(t_{0}\right)$, we further re-write

$$
\begin{aligned}
J= & x_{\mathrm{cn}}\left(t_{0}\right)^{\prime} M_{22}^{q} x_{\mathrm{cn}}\left(t_{0}\right)+x_{\mathrm{cn}}\left(t_{0}\right)^{\prime} \\
& \left(2\left(\left(M_{12}^{q}\right)^{\prime} x_{\mathrm{pl}}\left(t_{0}\right)+M_{23}^{q} x_{\mathrm{copy}}\left(t_{0}\right)-g_{2}^{q}\right)+* .\right.
\end{aligned}
$$

Since $M_{22}^{q}$ is positive semi-definite, (19) is convex on $x_{\mathrm{cn}}\left(t_{0}\right)$ and any vector $x_{\mathrm{cn}}^{*}\left(t_{0}\right)$ satisfying the first order condition

$$
M_{22}^{q} x_{\mathrm{cn}}^{*}\left(t_{0}\right)=\frac{1}{2} g_{2}^{q}-\left(M_{12}^{q}\right)^{\prime} x_{\mathrm{pl}}\left(t_{0}\right)-M_{23}^{q} x_{\text {copy }}\left(t_{0}\right)
$$

provides a global minimum to $J$ [15]. In general, (20) may not be solvable, but in our specific problem it can be proved that it always is (see appendix) and the minimum norm solution to (20) is given by (15).

\section{B. Choice of the reset map}

Since the optimal value for $x_{\mathrm{cn}}^{*}\left(t_{0}\right)$ in (15) depends on the process state $x_{\mathrm{pl}}\left(t_{0}\right)$ that is generally not accessible, we cannot directly use the expression in (15) to define the optimal reset map in (11). However, as mentioned in Section II, the component $x_{\text {copy }}(t)$ of the multicontroller state converges exponentially fast to the process state $x_{\mathrm{pl}}(t)$, for every control input $u(t)$. If we then replace $x_{\mathrm{pl}}\left(t_{0}\right)$ by $x_{\text {copy }}\left(t_{0}\right)$ in (15), we obtain an "asymptotically correct" minimum to (12), which justifies the following reset map

$$
\begin{aligned}
& F\left(x_{\text {mult }}\left(t_{0}^{-}\right), p, q, r\left(t_{0}\right)\right):=V_{1}^{q} \Lambda_{q}^{-1}\left(U_{1}^{q}\right)^{\prime} . \\
& \quad\left(\frac{1}{2} g_{2}^{q}-\left(M_{12}^{q}\right)^{\prime} x_{\text {copy }}\left(t_{0}^{-}\right)-M_{23}^{q} x_{\text {copy }}\left(t_{0}^{-}\right)\right) .
\end{aligned}
$$

Since $F(\cdot)$ is a function of the "past" value of $x_{\text {mult }}$, the right-hand side of (21) must only depend on the "past" value of $x_{\text {copy }}$. However, this is not a problem because $x_{\text {copy }}\left(t_{0}\right)=$ $x_{\text {copy }}\left(t_{0}^{-}\right)$, in view of (10).

\section{STABLE RESET MAP}

Although the reset map (21) minimizes the criteria (12), it may not necessarily result in a stable switched system. This is mainly because the optimization assumed that no further switching would occur in the interval $\left[t_{0}, t_{1}\right]$.

If one were willing to exclude the possibility of consecutive switching times separated by less than a given positive constant $\tau_{D}-$ a condition often referred to as $d w e l l$ time switching - then it would be possible to compute a sufficiently large $\tau_{D}$ for which the switched system (9)-(10) would be stable. However, here we do not want to make such an assumption on $\sigma(t)$. Instead, we want to appropriately modify the reset-map so as to guarantee stability for every piecewise continuous switching signal $\sigma(t)$, without significantly compromising the optimality of the reset map.

Suppose as in Section III that at a time $t=t_{0}$ the switching signal jumps from $\sigma\left(t_{0}^{-}\right)=p$ to $\sigma\left(t_{0}\right)=q$. We will show that stability under arbitrary switching can be achieved if we require that, at the switching time $t_{0}$, there should be no increase in the distance between $x_{\mathrm{cn}}$ and its steady-state value obtained from (13). Formally, this can be expressed as follows

$$
\left\|x_{\mathrm{cn}}\left(t_{0}\right)-K_{q} r\left(t_{0}\right)\right\|^{2} \leq\left\|x_{\mathrm{cn}}\left(t_{0}^{-}\right)-K_{q} r\left(t_{0}\right)\right\|^{2},
$$

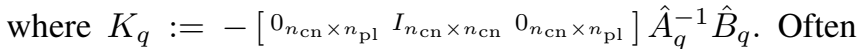
this constraint will be satisfied by choosing the global minimum to (12) with norm closest to $K_{q} r\left(t_{0}\right)$, which is given by

$$
\begin{aligned}
& x_{\mathrm{cn}}^{*}\left(t_{0}\right)=V_{1}^{q} \Lambda_{q}^{-1}\left(U_{1}^{q}\right)^{\prime} . \\
& \cdot\left(\frac{1}{2} g_{2}^{q}-\left(M_{12}^{q}\right)^{\prime} x_{\mathrm{pl}}\left(t^{-}\right)-M_{23}^{q} x_{\mathrm{copy}}\left(t^{-}\right)\right)+ \\
&+V_{2}^{q}\left(V_{2}^{q}\right)^{\prime} K_{q} r\left(t_{0}\right) .
\end{aligned}
$$

When compared with (15), (23) includes the additional term $V_{2}^{q}\left(V_{2}^{q}\right)^{\prime} K_{q} r\left(t_{0}\right)$, which is the projection of $K_{q} r\left(t_{0}\right)$ into the kernel of $M_{22}^{q}$. Therefore (23) still satisfies the first-order condition (20).

When (23) does not satisfy (22), we will need to find the minimum of (12) subject to the quadratic inequality (22). 
In view of (19), this is a convex quadratic optimization, subject to a convex quadratic constraint, which can be solved numerically very efficiently. As opposed to when (23) already satisfies (22), in this case the constraint (22) may lead to some increase in the value of the criteria (12).

\section{Stability}

The switched system (9)-(10) is said to be uniformly inputto-state stable (ISS) for a smooth input $r(t)$ if there exist constants $\lambda, c_{0}, c_{1}, c_{2}>0$ such that for every differentiable $r(t)$ and every piecewise constant switching signal $\sigma(t)$, the following inequality holds along solutions to (9)-(10):

$$
\begin{aligned}
\|x(t)\| \leq c_{0} e^{-\lambda t}\|x(0)\| \\
+c_{1} \sup _{\tau \in[0, t)}\|r(\tau)\|+c_{2} \sup _{\tau \in[0, t)}\|\dot{r}(\tau)\| .
\end{aligned}
$$

The following result confirms that the constraint (22) does guarantee closed-loop stability.

Theorem 2: The switched system (9)-(10) is uniformly ISS for a smooth input $r$ if the reset map satisfies the following inequality for every $p, q \in \mathcal{P}$, every $x_{\text {mult }}:=$ $\left[x_{\text {cn }}^{\prime} x_{\text {copy }}^{\prime}\right]^{\prime}$, and every $r_{0}$ :

$$
\left\|F\left(x_{\text {mult }}, p, q, r_{0}\right)-K_{q} r_{0}\right\|^{2} \leq\left\|x_{\mathrm{cn}}-K_{q} r_{0}\right\|^{2} .
$$

Proof of Theorem 2. Consider the signal $e(t)$ defined by

$$
e(t)=r(t)-y(t)+C x_{\text {copy }}(t), \quad \forall t \geq 0 .
$$

Because of (8) and the fact that the process is asymptotically stable, we conclude that $x_{\text {copy }}(t)$ converges exponentially fast to the process state $x_{\mathrm{pl}}(t)$. Consequently $C x_{\text {copy }}(t)$ converges to the process output $y(t)$ and we have that

$$
\|e(t)-r(t)\| \leq d e^{-\gamma t}\|x(0)\|,
$$

for appropriate positive constants $d, \gamma>0$. One should emphasize that (27) holds regardless of the control input $u(t)$, the switching signal $\sigma(t)$, and any resets to $x_{\mathrm{cn}}(t)$.

The importance of the signal $e(t)$ defined in (26) stems from the fact that, along solutions to (9)-(10), the state $x_{\mathrm{cn}}(t)$ evolves according to

$$
\dot{x}_{\mathrm{cn}}(t)=A_{\sigma(t)} x_{\mathrm{cn}}(t)+B_{\sigma(t)} e(t)
$$

on any time interval on which $\sigma(t)$ remains constant and

$$
x_{\mathrm{cn}}(t)=F\left(x_{\text {mult }}\left(t^{-}\right), \sigma\left(t^{-}\right), \sigma(t), r(t)\right),
$$

at every switching time $t$. Suppose now that we define

$$
v(t):=\left\|x_{\mathrm{cn}}(t)-K_{\sigma(t)} r(t)\right\|^{2} .
$$

On any interval on which $\sigma(t)$ remains constant equal to some $p \in \mathcal{P}$, we have that

$$
\begin{aligned}
\dot{v}= & x_{\mathrm{cn}}(t)^{\prime}\left(A_{p}+A_{p}^{\prime}\right) x_{\mathrm{cn}}(t) \\
& +2 x_{\mathrm{cn}}(t)^{\prime}\left(B_{p} e(t)-K_{p} \dot{r}(t)-A_{p}^{\prime} K_{p} r(t)\right) \\
& -2 r(t)^{\prime} K_{p}^{\prime}\left(B_{p} e(t)-K_{p} \dot{r}(t)\right) .
\end{aligned}
$$

Because of (4) and using fairly standard square completion arguments, it is possible to find a sufficiently small constant $\mu$ and sufficiently large constants $d_{1}, d_{2}, d_{3}$ (independent of the value of $p$ ) such that

$$
\dot{v}(t) \leq-\mu v(t)+d_{1}\|e(t)\|^{2}+d_{2}\|r(t)\|^{2}+d_{3}\|\dot{r}(t)\|^{2} .
$$

Since $v(t)$ does not increase at switching times because of (25), we conclude that

$$
\begin{array}{rl}
v(t) \leq e^{-\mu t} & v(0)+d_{1} \int_{0}^{t} e^{-\mu(t-\tau)}\|e(\tau)\|^{2} d \tau \\
& +\frac{d_{2}}{\mu} \sup _{\tau \in[0, \tau)}\|r(\tau)\|^{2}+\frac{d_{3}}{\mu} \sup _{\tau \in[0, \tau)}\|\dot{r}(\tau)\|^{2} .
\end{array}
$$

From this and (27), we conclude that $\left\|x_{\mathrm{cn}}(t)\right\|$ satisfies an inequality like (24). It remains to show that the remaining components $x_{\mathrm{pl}}(t)$ and $x_{\text {copy }}(t)$ of the overall state $x(t)$ also satisfy such inequalities. To this effect note that the input $u(t)$ to the process and to the system (8) can be written as

$$
u(t)=C_{\sigma(t)} x_{\mathrm{cn}}(t)+D_{\sigma(t)} e(t)
$$

[cf. (1), (5)-(6), and (26)] and therefore $u(t)$ also satisfies an inequality like (24). Finally, since $x_{\mathrm{pl}}(t)$ and $x_{\text {copy }}(t)$ are the states of asymptotically stable LTI systems driven by $u(t)$, these states also satisfy an inequality like (24).

\section{Simulation Results}

In this section we compare the transients due to switching for the multicontrollers proposed here, in [4], and in [3].

Consider a process with transfer function $g(s)=\frac{10}{s+1}$ and two controllers with transfer functions given by (3) with

$q_{1}(s)=\frac{2 s^{2}+5 s+3}{100 s^{2}+120 s+30}, \quad q_{2}(s)=\frac{6 s^{2}+46 s+40}{100 s^{2}+160 s+400}$ among which one would like to switch. This process and controller transfer functions appeared in [4]. The subsequent figures show the output and reference signals of the resulting closed-loop switched system. In Figure 4 the blue dotted lines show the output $y(t)$ of the system for the controller switching strategy suggested in [4], whereas the red solid lines show $y(t)$ for the multicontroller proposed here. Figure 5 compares the multicontroller proposed here with the two solutions proposed in [3], which correspond to (i) a reset to zero of $x_{\mathrm{cn}}(t)$ at every switching and (ii) maintaining $x_{\mathrm{cn}}(t)$ continuous at every switching instant. In all simulations the use of the multicontroller proposed here, resulted in a significant performance improvement.

It is worth to notice that in all the simulations presented here, the global optimal reset values given by (23) automatically satisfied the constraint (22). In fact, this was the case in every simulation that we encountered.

\section{CONCLUSION AND FUTURE WORKS}

When switching between a given family of LTI controllers, significant performance improvements can be obtained by appropriate selection of controller realizations and controller state resets at switching times. The controller realizations proposed are inspired by the results in [3] and the state resets are chosen so as to minimize a quadratic cost function. By 


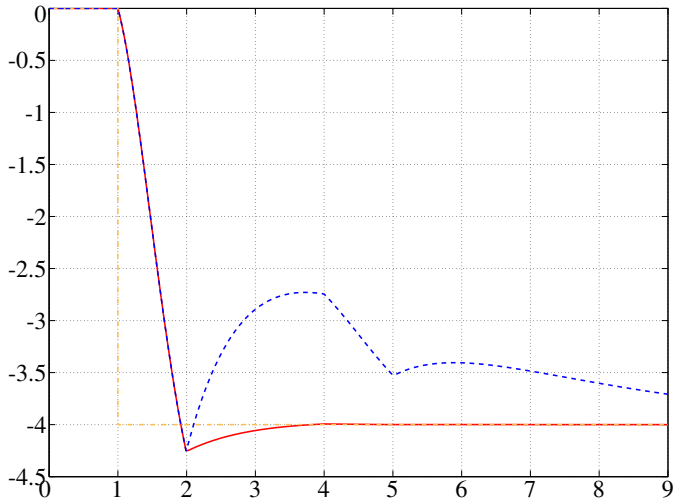

(a) Switching times: 2, 4, and $5 \mathrm{sec}$

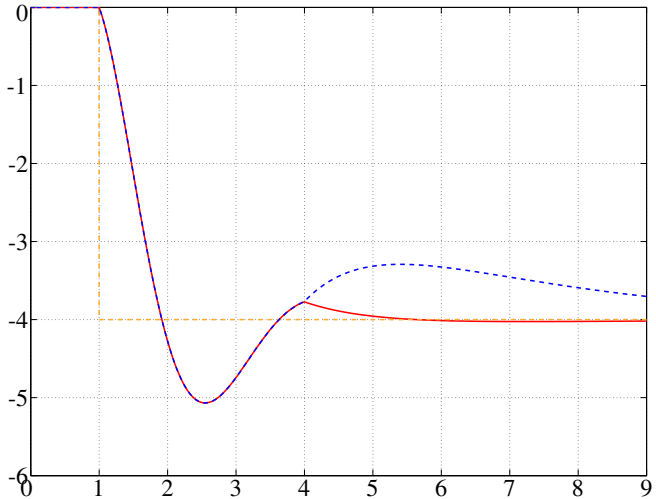

(b) Switching time: $4 \mathrm{sec}$

Fig. 4. Transient responses for the multicontroller proposed here (red solid line) and for the multicontroller proposed in [4] (blue dotted line).

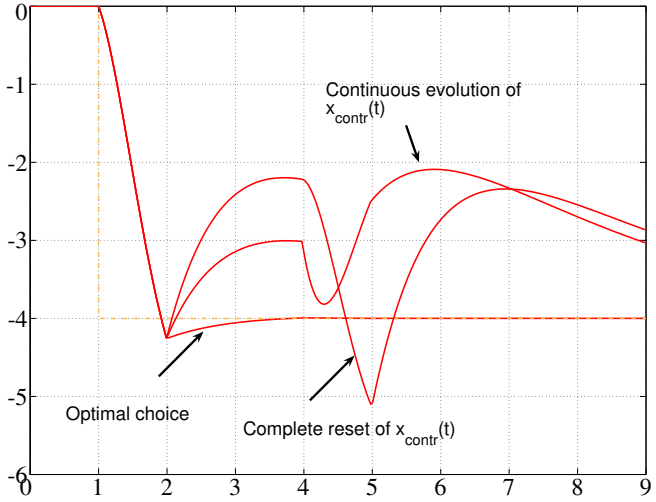

Fig. 5. Transient responses for the multicontroller proposed here and for the two alternative multicontrollers proposed in [3]. The plot shows the transients due to three control switchings at times 2,4 , and $5 \mathrm{sec}$.

introducing a norm-constraint in the state-reset optimization, we are able to guarantee that the closed-loop switched system remains stable for arbitrary switching.

In all our simulations, the constraint introduced to guarantee stability was never active, which leads us to conjecture that this constraint may not be needed, at least for certain classes of processes/controllers. Verifying this conjecture remains a topic for future research. In this paper, we assumed that the switching signal $\sigma(t)$ was provided externally. However, the minimization of $J$ in (12) could also provide a valid criteria for selecting appropriate switching times. This is another topic for future research.

\section{APPENDIX}

Proof that (20) is always solvable. Let $w:=\frac{1}{2} g_{2}^{q}-$ $\left(M_{12}^{q}\right)^{\prime} x_{\mathrm{pl}}\left(t_{0}\right)-M_{23}^{q} x_{\text {copy }}\left(t_{0}\right)$ and assume by contradiction that there is no solution $x_{\mathrm{cn}}^{*}\left(t_{0}\right)$ to the equation $M_{22}^{q} x_{\mathrm{cn}}^{*}\left(t_{0}\right)=w$, which means that $w \notin \operatorname{Im} M_{22}^{q}=$ $\left(\operatorname{Ker} M_{22}^{q}\right)^{\perp}$, where $\left(\operatorname{Ker} M_{22}^{q}\right)^{\perp}$ denotes the orthogonal complement of $\operatorname{Ker} M_{22}^{q}$. In this case, there must exist a vector $z \in \operatorname{Ker} M_{22}^{q}$ such that $w^{\prime} z \neq 0$. If we then set $x_{\mathrm{cn}}\left(t_{0}\right)=\alpha z$ in (19), we obtain $J=-2 \alpha w^{\prime} z+*$. However, this would lead to a negative value for $J$ for $\alpha$ sufficiently large, which leads to a contradiction since $J \geq 0$.

\section{REFERENCES}

[1] Boyd, S. P., \& Barrat, C. H., Linear controller design: Limits of performance, New Jersey: Prentice Hall, 1991.

[2] Hespanha, J. P., \& Morse, A. S., Towards the high performance control of uncertain process via supervision, in Proc. of the 30th annual conf. on information sciences and systems 1, 405-410, 1996.

[3] Hespanha, J. P., \& Morse, A. S., Switching between stabilizing controllers, Automatica 38 (2002), 1905-1917.

[4] Stewart, G. E. \& Dumont, G. A., Finite Horizon Based Switching Between Stabilizing Controllers, in Proc. American Control Conference, 1550-1556, 2006.

[5] Liberzon, D., Switching in Systems and Control, Birkhauser, Boston, 2003.

[6] Lin, H., \& Antsaklis, P. J., Stability and stabilizability of Switched Linear Systems: a short survey of recent results, in Proc. of the 2005 IEEE Int. Symp. on intelligent Control, Limassul, Cyprus, 24-29, 2005.

[7] De Carlo, R.A., Branicky, M.S., Petterson, S. \& Lennartson, B., Perspectives and results on the stabilizability of hybrid systems, in Proc. of the IEEE, 88(7), 1069-1082, 2000.

[8] Liberzon, D. \& Morse, A.S., Basic problems in stability and design of switched systems, in IEEE Control System Mag., 19(5), 59-70, 1999.

[9] Bemporad, A., Borrelli, F., Morari, M., Piecewise linear optimal controllers for hybrid systems, in Proc. American Control Conference, $1190-1194,2000$

[10] Xu, X. \& Antsaklis, P.J., Optimal control of switched systems via nonlinear optimization based on direct differentiation of value functions, in Int. Journal of Control, 75, 1406-1426, 2002.

[11] Azuma, S., Egerstedt. M. \& Wardi, Y., Output-based optimal timing control of switched systems, in Hybrid Systems: Computation and Control, Springer-Verlag, pp. 64-78, Santa Barbara, CA, 2006.

[12] Piccoli, B., Hybrid systems and optimal control, in Proc. 37th IEEE Conference on Decision and Control, 13-18, 1998.

[13] Xu, X. \& Antsaklis, P.J., Optimal control of switched systems based on parametrization of the switching instants, IEEE Transaction on Automatic Control, 49(1), (2004), 2-16.

[14] Sun, Z., Ge, S.S., Switched Linear Systems: control and design, Springer, 2005.

[15] Boyd, S., Vandenberghe, L., Convex Optimization, Cambridge University Press, 2004. 